\title{
MULTIPARAMETER POINTWISE ERGODIC THEOREMS FOR MARKOV OPERATORS ON $L_{\infty}$
}

\author{
RYotaRo SATO
}

\begin{abstract}
Let $P_{1}, \ldots, P_{d}$ be commuting Markov operators on $L_{\infty}(X, \mathcal{F}, \mu)$, where $(X, \mathcal{F}, \mu)$ is a probability measure space. Assuming that each $P_{i}$ is either conservative or invertible, we prove that for every $f$ in $L_{p}(X, \mathcal{F}, \mu)$ with $1 \leq p<\infty$ the averages

$$
A_{n} f=(n+1)^{-d} \sum_{0 \leq n_{i} \leq n} P_{1}^{n_{1}} P_{2}^{n_{2}} \ldots P_{d}^{n_{d}} f \quad(n \geq 0)
$$

converge almost everywhere if and only if there exists an invariant and equivalent finite measure $\lambda$ for which the Radon-Nikodym derivative $v=d \lambda / d \mu$ is in the dual space $L_{p^{\prime}}(X, \mathcal{F}, \mu)$. Next we study the case in which there exists $p_{1}$, with $1 \leq p_{1} \leq \infty$, such that for every $f$ in $L_{p}(X, \mathcal{F}, \mu)$ the limit function belongs to $L_{p_{1}}(X, \mathcal{F}, \mu)$. We give necessary and sufficient conditions for this problem.
\end{abstract}

\section{Introduction}

Let $(X, \mathcal{F}, \mu)$ be a probability measure space and let $P_{i}(i=1,2, \ldots, d)$ be commuting Markov operators defined on $L_{\infty}(X, \mathcal{F}, \mu)$. In this paper we assume that each $P_{i}$ is conservative or invertible, and prove that the averages

$$
A_{n} f=(n+1)^{-d} \sum_{0 \leq n_{i} \leq n} P_{1}^{n_{1}} P_{2}^{n_{2}} \ldots P_{d}^{n_{d}} f \quad(n \geq 0)
$$

converge almost everywhere for every $f$ in $L_{p}(X, \mathcal{F}, \mu), 1 \leq p<\infty$, if and only if there exists an invariant and equivalent finite measure $\lambda$ for which the Radon-Nikodym derivative $v=d \lambda / d \mu$ satisfies $v \in L_{p^{\prime}}(\mu)$ with $1 / p+1 / p^{\prime}=1$. This is a new result, even if $d=1$. Next we 
consider the question whether there exists a constant $p_{1}, 1 \leq p_{1} \leq \infty$, for which the limit functions belong to $L_{p_{1}}(X, \mathcal{F}, \mu)$. We give necessary and sufficient conditions for this problem.

Let $(X, \mathcal{F}, \mu)$ be a probability measure space. By a Markov operator $P$ defined on $L_{\infty}(\mu)=L_{\infty}(X, \mathcal{F}, \mu)$ we mean a linear operator from $L_{\infty}(\mu)$ into itself such that

(i) $P$ is positive: $P f \geq 0$ whenever $f \in L_{\infty}^{+}(\mu)$,

(ii) $P$ is a contraction: $\|P\|_{\infty} \leq 1$,

(iii) $\lim _{n} P f_{n}=0$ a.e. on $X$ whenever $f_{n} \in L_{\infty}^{+}(\mu)$ and $f_{n} \downarrow 0$ a.e. on $X$.

By virtue of (iii) and the Radon-Nikodym theorem we may define an operator on $L_{1}(\mu)=L_{1}(X, \mathcal{F}, \mu)$ by the relation

$$
\int_{B} u P d \mu=\int_{X} u\left(P \mathbf{1}_{B}\right) d \mu \quad\left(u \in L_{1}(\mu) \text { and } B \in \mathcal{F}\right),
$$

$\mathbf{1}_{B}$ being the indicator function of $B$. Here we use the same symbol $P$ for the $L_{1}(\mu)$ operator, but we write it to the right of its variable. $P$ is called invertible if $P \mathbf{1}=1$ and $P$ is a one-to-one onto mapping of $L_{\infty}(\mu)$, and conservative if $f \in L_{\infty}^{+}(\mu)$ and $P f \leq f$ imply $P f=f$. It is known (see e.g. [4]) that $P$ is conservative if and only if $\sum_{i-0}^{\infty} u P^{i}=\infty$ a.e. on $X$ whenever $u \in L_{1}^{+}(\mu)$ and $u>0$ a.e. on $X$. Since $P$ is positive, we may extend the domain of $P$ to the space $M^{+}(\mu)=M^{+}(X, \mathcal{F}, \mu)$ of all nonnegative extended real valued measurable functions on $X$, by the relations

$$
P f=\lim _{n} P f_{n} \text { a.e. where } f_{n} \in L_{\infty}^{+}(\mu) \text { and } f_{n} \uparrow f \text { on } X
$$

and

$$
u P=\lim _{n} u_{n} P \text { a.e. where } u_{n} \in L_{1}^{+}(\mu) \text { and } u_{n} \uparrow u \text { on } X \text {. }
$$

It is easily seen that by this process $P f$ and $u P$ are uniquely determined a.e. on $X$. We then have

$$
\langle u P, f\rangle=\int_{X}(u P) f d \mu=\int_{X} u(P f) d \mu=\langle u, P f\rangle .
$$

In this paper we consider several commuting Markov operators $P_{i}(i=$ $1,2, \ldots, d)$ defined on $L_{\infty}(\mu)$. Throughout the paper we will assume that 
each $P_{i}$ is conservative or invertible. Associated with these operators we define the averages

$$
A_{n} f=(n+1)^{-d} \sum_{0 \leq n_{i} \leq n} P_{1}^{n_{1}} P_{2}^{n_{2}} \ldots P_{d}^{n_{d}} f \quad(n \geq 0)
$$

and the maximal operator

$$
M f=\sup _{n \geq 0} A_{n}|f| .
$$

Also we define the $\sigma$-field of all invariant subsets of $X$ :

$$
\mathcal{I}=\left\{B \in \mathcal{F}: P_{i} \mathbf{1}_{B}=\mathbf{1}_{B} \text { for all } i=1,2, \ldots, d\right\} .
$$

We call the system $\left\{P_{1}, P_{2}, \ldots, P_{d}\right\}$ ergodic if $\mathcal{I}$ is the trivial $\sigma$-field.

It is known that if $\mu$ is invariant under the $P_{i}$, i.e., $1 P_{i}=1$ for all $i=1,2, \ldots, d$ then the sequence $\left\{A_{n} f\right\}$ converges a.e. for every $f$ in $L_{p}^{+}(\mu), 1 \leq p \leq \infty$. But, if $\mu$ is not assumed to be invariant, we cannot expect the almost everywhere convergence of $\left\{A_{n} f\right\}$ for every $f$ in $L_{p}^{+}(\mu)$, $1 \leq p \leq \infty$. Therefore the author thinks that it would be of interest to characterize those probability measures $\mu$ for which the sequence $\left\{A_{n} f\right\}$ converges a.e. for every $f$ in $L_{p}^{+}(\mu), 1 \leq p \leq \infty$. As is easily seen, such a characterization for $p=\infty$ is the existence of an invariant and equivalent finite measure. Thus we will concern ourselves with the case $1 \leq p<\infty$ below. It is interesting to note here that this problem for $d$-parameter groups of null preserving point transformations was recently examined by Martín-Reyes [5]. Hence our results may be regarded as generalizations (and improvements) of those due to Martín-Reyes. We will give a new characterization which has a connection with the invariant measure problem. That is, we will prove in Section 3 that the sequence $\left\{A_{n} f\right\}$ converges a.e. for every $f$ in $L_{p}^{+}(\mu)$ if and only if there exists an invariant and equivalent finite measure $\lambda$ for which the Radon-Nikodym derivative $v=d \lambda / d \mu$ satisfies

$$
v \in L_{p^{\prime}}^{+}(X, \mathcal{F}, \mu) \text { with } 1 / p+1 / p^{\prime}=1 .
$$

Next, let us suppose that $f^{*}(x)=\lim _{n} A_{n} f(x)$ exists a.e. for all $f$ in $L_{p}^{+}(\mu), 1 \leq p \leq \infty$. Do the functions $f^{*}$ belong to $L_{p}^{+}(\mu)$ ? As is easily seen, this is not true in general, unless $p=\infty$. So the following question arises naturally. Does there exist a constant $p_{1}, 1 \leq p_{1} \leq \infty$, such that $f^{*} \in L_{p_{1}}^{+}(\mu)$ for all $f \in L_{p}^{+}(\mu)$ ? We will prove in Section 3 that the limit $f^{*}=\lim _{n} A_{n} f$ exists a.e. and $f^{*} \in L_{p_{1}}^{+}(\mu)$ for all $f$ in $L_{p}^{+}(\mu)$ if and only 
if for any $u \in L_{p_{1}^{\prime}}(\mu), 1 / p_{1}+1 / p_{1}^{\prime}=1$, the limit $u_{0}^{*}=\lim _{n} u A_{n}$ exists a.e and in the norm topology of $L_{1}(\mu)$ and further we have $u_{0}^{*} \in L_{p^{\prime}}(\mu)$, $1 / p+1 / p^{\prime}=1$.

In Section 2 we study the special case where $\mu$ is invariant under the $P_{i}$, and consider the measure $\lambda=V d \mu$, where $V$ is a positive measurable function on $X$. Regarding the $P_{i}$ as commuting Markov operators defined on $L_{\infty}(X, \mathcal{F}, V d \mu)$, we obtain some preliminary results which may be of independent interest by themselves.

In what follows two functions $f$ and $g$ are not distinguished provided that $f=g$ a.e. on $X$, and if $1 \leq p \leq \infty$ then $p^{\prime}$ will be its conjugate exponent, i.e. $1 / p+1 / p^{\prime}=1$.

\section{The invariant measure case}

In this section we assume that the probability measure $\mu$ is invariant under the $P_{i}$, or equivalently that $1 P_{i}=1=P_{i} \mathbf{1}$ for all $i=1,2, \ldots, d$, and consider the measure $\lambda=V d \mu$, where $V$ is a positive measurable function on $X$. It may happen that $\lambda X=\int_{X} V d \mu=\infty$.

Theorem 2.1. Let $P_{i}(1 \leq i \leq d)$ be commuting Markov operators on $L_{\infty}(X, \mathcal{F}, \mu)$, where $(X, \mathcal{F}, \mu)$ is a probability measure space. Assume that $\mu$ is invariant under the $P_{i}$. Let $V$ be a positive measurable function on $X$, and let $1 \leq p<\infty$. Then the following are equivalent.

(a) For every $f$ in $L_{p}^{+}(V d \mu), \lim _{n} A_{n} f$ exists and is finite a.e. on $X$.

(b) For every $f$ in $L_{p}^{+}(V d \mu), M f<\infty$ a.e. on $X$.

(c) There exists a positive measurable function $U$ on $X$ such that

$$
\int_{\{M f>t\}} U d \mu \leq t^{-p} \int_{X} f^{p} V d \mu \quad\left(t>0, f \in L_{p}^{+}(V d \mu)\right) .
$$

(d) There exists a positive measurable function $U$ on $X$ such that

$$
\liminf _{n} \int_{\left\{A_{n} f>t\right\}} U d \mu \leq t^{-p} \int_{X} f^{p} V d \mu \quad\left(t>0, f \in L_{p}^{+}(V d \mu)\right) .
$$

(e) $\operatorname{ess} \sup \left\{W \in M^{+}(X, \mathcal{I}, \mu): W \leq V\right\}>0$ a.e. on $X$ (if $p=1$ ); $E\left\{V^{1-p^{\prime}} \mid \mathcal{I}\right\}<\infty$ a.e. on $X$ (if $1<p<\infty$ ).

Proof: It is clear that $(\mathrm{a}) \Rightarrow(\mathrm{b})$ and $(\mathrm{c}) \Rightarrow(\mathrm{d})$.

(b) $\Rightarrow$ (c). (b) implies that for each $n \geq 0$ and all $f \in L_{p}^{+}(V d \mu)$, $\left|A_{n} f\right|<\infty$ a.e. on $X$. Thus the operator $A_{n}$ can be considered to be a 
continuous mapping from $L_{p}(V d \mu)$ to $L_{0}(\mu)$, where $L_{0}(\mu)$ denotes the space of all finite valued measurable functions on $X$, equipped with the topology of the convergence in measure. By this and Banach's principle (cf. e.g. $[3$, p. 2]) we see that the sublinear operator $f \rightarrow M|f|$ is continuous from $L_{p}(V d \mu)$ to $L_{0}(\mu)$. Hence (c) follows from Nikishin's theorem (cf. [2, p. 536]).

(d) $\Rightarrow$ (a). Let $f \in L_{p}^{+}(V d \mu)$, and choose $f_{N} \in L_{1}^{+}(\mu), N=1,2, \ldots$, so that $f_{N} \uparrow f$ on $X$. Since $\mu$ is invariant under the $P_{i}$, it follows that $\left\|P_{i}\right\|_{r}=1$ for all $1 \leq r \leq \infty$, and hence the classical pointwise ergodic theorem for $d$-parameter semigroups of Dunford-Schwartz operators can be applied to infer that

$$
\lim _{n} A_{n} f_{N}=E\left\{f_{N} \mid \mathcal{I}\right\} \text { a.e. on } X \text {. }
$$

Using this we see that the pointwise limit $f^{*}(x)=\lim _{n} A_{n} f(x)$ exists a.e. on $X$ (but may equal to infinity on some subset of $X$ ) and that

$$
f^{*}=E\{f \mid \mathcal{I}\} \text { a.e. on } X \text {. }
$$

To prove that $f^{*}<\infty$ a.e. on $X$, let us write $B=\left\{x: f^{*}(x)=\infty\right\}$. Then, since $B \subset \liminf _{n}\left\{A_{n} f>t\right\}$ for any $t>0$, we have by Fatou's lemma and (d),

$$
\int_{B} U d \mu \leq \liminf _{n} \int_{\left\{A_{n} f>t\right\}} U d \mu \leq t^{-p} \int_{X} f^{p} V d \mu .
$$

Letting $t \uparrow \infty$, it follows that $\int_{B} U d \mu=0$, and consequently $\mu B=0$.

(a) $\Rightarrow$ (e). As in [8], we use the ergodic decomposition technique. We first note that for the proof it may be assumed without loss of generality that $(X, \mathcal{F}, \mu)$ is a Lebesgue measure space in the sense of Rokhlin [6]. Then using Rokhlin's theory we can find a countable family $\left\{E_{i}\right\}$ of sets in $\mathcal{I}$ such that if $\xi$ denotes the decomposition of $X$ induced by $\left\{E_{i}\right\}$, i.e., $C \in \xi$ has the form

$$
C=\bigcap_{i} E_{i}\left(\varepsilon_{i}\right)
$$

where $\varepsilon_{i}= \pm 1, E_{i}(1)=E_{i}$ and $E_{i}(-1)=X \backslash E_{i}$, then:

(i) The factor space $\left(X / \xi, \mathcal{F}_{\xi}, \mu_{\xi}\right)$ of $(X, \mathcal{F}, \mu)$ with respect to $\xi$ is a Lebesgue measure space.

(ii) To a.e. $C \in X / \xi$ with respect to $\mu_{\xi}$ there corresponds a Lebesgue measure $\mu_{C}$ on $C$ such that if $B \in \mathcal{F}$ then $B \cap C$ is measurable with 
respect to $\mu_{C}$ for a.e. $C \in X / \xi$, and the function $h(C)=\mu_{C}(B \cap C)$ is measurable with respect to $\mu_{\xi}$ and satisfies, for all $Z \in \mathcal{F}$ of the form $Z=\iota^{-1}(Z / \xi)$, where $\iota: X \rightarrow X / \xi$ denotes the canonical mapping,

$$
\mu(B \cap Z)=\int_{Z / \xi} h(C) d \mu_{\xi}(C)=\int_{Z / \xi} \mu_{C}(B \cap C) d \mu_{\xi}(C) .
$$

(iii) To a.e. $C \in X / \xi$ there correspond commuting Markov operators $P(C)_{1}, P(C)_{2}, \ldots, P(C)_{d}$ defined on $L_{\infty}\left(C, \mu_{C}\right)$ such that the system $\left\{P(C)_{1}, P(C)_{2}, \ldots, P(C)_{d}\right\}$ is ergodic, and also such that if $u \in L_{1}(X, \mathcal{F}, \mu)$ and $f \in L_{\infty}(X, \mathcal{F}, \mu)$ then, for a.e. $C \in X / \xi$,

$$
u_{C} P(C)_{i}=\left(u P_{i}\right)_{C} \text { and } P(C)_{i} f_{C}=\left(P_{i} f\right)_{C} \text { for all } i=1,2, \ldots, d,
$$

where $u_{C},\left(u P_{i}\right)_{C}, f_{C}$ and $\left(P_{i} f\right)_{C}$ denote, respectively, the restriction functions of $u, u P_{i}, f$ and $P_{i} f$ to the set $C$.

To prove the implication (a) $\Rightarrow$ (e) for $p=1$ we define

$$
\tilde{V}=\operatorname{ess} \sup \left\{W \in M^{+}(X, \mathcal{I}, \mu): W \leq V\right\}
$$

and assuming that $\mu\{\tilde{V}=0\}>0$, we derive a contradiction as follows. Since $\{\tilde{V}=0\} \in \mathcal{I}$, it may be supposed without loss of generality that $\{\tilde{V}=0\}=X$. Then we use the ergodic decomposition technique. Define the funciton $h_{n}$ on $X / \xi$ by

$$
h_{n}(C)=\mu_{C}(\{V<1 / n\} \cap C) \quad(n \geq 1) .
$$

Since $h_{n}(C)>0$ for a.e. $C \in X / \xi$ because $\tilde{V}=0$ on $X$, if $f_{n}$ denotes the function on $X$ defined by

$$
f_{n}(x)=n^{-1}\left(h_{n}(C)\right)^{-1} \mathbf{1}_{\{V<1 / n\}}(x) \quad(x \in C \in X / \xi),
$$

then we have

$$
\int_{C} f_{n} d \mu_{C}=1 / n \quad(C \in X / \xi)
$$

and

$$
\begin{aligned}
\int_{X} f_{n} V d \mu & =\int_{X / \xi}\left(\int_{C} f_{n} V d \mu C\right) d \mu_{\xi}(C) \\
& \leq \int_{X / \xi}\left(1 / n^{2}\right) d \mu_{\xi}(C)=1 / n^{2} .
\end{aligned}
$$


Therefore the function $f=\sum_{n=1}^{\infty} f_{n}$ satisfies $f \in L_{1}^{+}(V d \mu)$. But, since $f_{C} \notin L_{1}^{+}\left(\mu_{C}\right)$ for a.e. $C \in X / \xi$, it follows from (iii) and the classical pointwise ergodic theorem for $d$-parameter semigroups of DunfordSchwarz operators that for a.e. $C \in X / \xi$,

$$
f^{*}(x)=\lim _{n} A_{n} f(x)=\infty \text { a.e. on } C
$$

with respect to the measure $\mu_{C}$. It follows that $f^{*}(x)=\infty$ a.e. on $X$ with respect to the measure $\mu$. This contradicts (a).

Next, let us consider the case $1<p<\infty$, and suppose that the set

$$
B=\left\{x: E\left\{V^{1-p^{\prime}} \mid \mathcal{I}\right\}(x)=\infty\right\}
$$

is not a null set. Since $B \in \mathcal{I}$, we then suppose without loss of generality that $B=X$, and from this we derive a contradiction as follows. First we note that if $E\left\{V^{1-p^{\prime}} \mid \mathcal{I}\right\}=\infty$ a.e. on $X$ then

$$
\int_{C} V^{1-p^{\prime}} d \mu_{C}=\infty \text { for a.e. } C \in X / \xi .
$$

Using this and doing as in the proof of the implication (a) $\Rightarrow(b)$ of Theorem 1 in [8] (see especially p. 75 in [8]) it is possible to construct a function $f$ in $L_{p}^{+}(V d \mu)$ so that for a.e. $C \in F / \xi$, where $F$ is a set in $\mathcal{I}$ and satisfies $\mu F>0$, we have $\int_{C} f d \mu_{C}=\infty$. Then it follows from (iii) that for a.e. $C \in F / \xi$,

$$
f^{*}(x)=\lim _{n} A_{n} f(x)=\infty \text { a.e. on } C
$$

with respect to the measure $\mu_{C}$. Hence $f^{*}(x)=\infty$ a.e. on $F$ with respect to the measure $\mu$. This contradicts (a), because $f \in L_{p}^{+}(V d \mu)$.

(e) $\Rightarrow$ (a). (e) implies the existence of a sequence $\left\{X_{N}\right\}$ of sets in $\mathcal{I}$, with $X_{N} \uparrow X$, such that if $V_{N}$ denotes the restriction function of $V$ to $X_{N}$ then

$$
V_{N}^{-1} \in L_{p^{\prime}}^{+}\left(X_{N}, V d \mu\right) .
$$

Here, since $X_{N} \in \mathcal{I}$, for the proof of (a) it may be supposed without loss of generality that $X_{N}=X$. Then for any $f \in L_{p}^{+}(V d \mu)$ the Hölder inequality yields

$$
\int_{X} f d \mu=\int_{X} f \frac{1}{V} V d \mu \leq \begin{cases}\left(\int_{X} f V d \mu\right)\left\|\frac{1}{V}\right\|_{L_{\infty}(X, V d \mu)}<\infty & (p=1) \\ \left(\int_{X} f^{p} V d \mu\right)^{1 / p}\left(\int_{X}\left(\frac{1}{V}\right)^{p^{\prime}} V d \mu\right)^{1 / p^{\prime}}<\infty \\ (1<p<\infty),\end{cases}
$$


so that $f \in L_{1}^{+}(\mu)$, and hence (a) follows from the classical pointwise ergodic theorem for $d$-parameter semigroups of Dunford-Schwartz operators. The proof is complete.

The following corollary is immediate from the equivalence of (a) and (e) in Theorem 2.1.

Corollary 2.2. Let $P_{i}(i=1,2, \ldots, d)$ be as in Theorem 2.1. Suppose in addition that the system $\left\{P_{1}, P_{2}, \ldots, P_{d}\right\}$ is ergodic. Then $\lim _{n} A_{n} f$ exists and is finite a.e. on $X$ for every $f$ in $L_{p}^{+}(V d \mu), 1 \leq p<\infty$, if and only if $V^{-1} \in L_{p^{\prime}}(X, \mathcal{F}, V d \mu)$.

\section{The general case}

Theorem 3.1. Let $(X, \mathcal{F}, \mu)$ be a probability measure space and let $P_{i}(i=1,2, \ldots, d)$ be commuting Markov operators on $L_{\infty}(X, \mathcal{F}, \mu)$. Assume that each $P_{i}$ is conservative or invertible. If $1 \leq p<\infty$, then the following are equivalent.

(a) For every $f$ in $L_{p}^{+}(\mu), \lim _{n} A_{n} f$ exists and is finite a.e. on $X$.

(b) For every $f$ in $L_{p}^{+}(\mu), M f<\infty$ a.e. on $X$.

(c) There exists a positive measurable function $U$ on $X$ such that

$$
\int_{\{M f>t\}} U d \mu \leq t^{-p} \int_{X} f^{p} d \mu \quad\left(t>0, f \in L_{p}^{+}(\mu)\right) .
$$

(d) There exist a positive measurable function $U$ on $X$, a positive constant $r$ and a subsequence $\{n(k)\}$ of the sequence $\{n\}$ such that

$$
\int_{\left\{A_{n(k)} f>t\right\}} U d \mu \leq t^{-r}\left(\int_{X} f^{p} d \mu\right)^{r / p} \quad\left(t>0, f \in L_{p}^{+}(\mu)\right) .
$$

(e) For every $u$ in $L_{1}(\mu)$, the sequence $\left\{u A_{n}\right\}$ converges in the norm topology of $L_{1}(\mu)$ and also a.e. on $X$; further to each $v \in L_{1}^{+}(\mu)$ with $v P_{i}=v$ for all $i=1,2, \ldots, d$ there corresponds a sequence $\left\{X_{N}\right\}$ of sets in $\mathcal{I}$ such that $X_{N} \uparrow X$ and the restriction function $v_{N}$ of $v$ to $X_{N}$ is in $L_{p^{\prime}}\left(X_{N}, \mu\right)$ for each $N \geq 1$.

(f) There exists $v \in L_{p^{\prime}}^{+}(\mu)$ with $v>0$ a.e. on $X$ and $v P_{i}=v$ for all $i=1,2, \ldots, d$.

In order to prove Theorem 3.1 we begin by proving the following 
Lemma 3.2. Let $P_{i}(i=1,2, \ldots, d)$ be commuting Markov operators on $L_{\infty}(X, \mathcal{F}, \mu)$, where $(X, \mathcal{F}, \mu)$ is a probability measure space. Assume that each $P_{i}$ is conservative or invertible. Then the sequence $\left\{A_{n} f\right\}$ converges a.e. on $X$ for every $f$ in $L_{\infty}(X, \mathcal{F}, \mu)$ if and only if there exists a function $v$ in $L_{1}^{+}(X, \mathcal{F}, \mu)$ such that $v>0$ a.e. on $X$ and $v P_{i}=v$ for all $i=1,2, \ldots, d$.

Proof: Suppose the first assertion of the lemma holds. Then for any $u \in L_{1}(\mu)$ and $f \in L_{\infty}(\mu)$ the sequence

$$
\left\langle u A_{n}, f\right\rangle=\int_{X}\left(u A_{n}\right) f d \mu=\int_{X} u\left(A_{n} f\right) d \mu=\left\langle u, A_{n} f\right\rangle
$$

converges to a finite limit as $n \rightarrow \infty$. It follows from the Vitali-HahnSaks theorem that $\left\{u A_{n}\right\}$ converges weakly in $L_{1}(\mu)$. Hence by a mean ergodic theorem (c.f. e.g. [4, Theorem 2.1.5]), $\left\{u A_{n}\right\}$ converges strongly in $L_{1}(\mu)$. Let $v \in L_{1}^{+}(\mu)$ be the limit function of the sequence $\left\{1 A_{n}\right\}$ in $L_{1}(\mu)$. Since $v P_{i}=v$ for each $i$, it follows that the set $B=\{x: v(x)=0\}$ satisfies $P_{i} 1_{B} \leq 1_{B}$ for each $i$. Here if $P_{i}$ is conservative, then we have $P_{i} \mathbf{1}_{B}=\mathbf{1}_{B}$. On the other hand, if $P_{i}$ is invertible then, since $v P_{i}=v=v P_{i}^{-1}$, we have $P_{i} \mathbf{1}_{B}=\mathbf{1}_{B}$, too. Consequently $B \in \mathcal{I}$, and thus

$$
\mu B=\left\langle 1, A_{n} \mathbf{1}_{B}\right\rangle=\left\langle 1 A_{n}, \mathbf{1}_{B}\right\rangle \rightarrow \int_{B} v d \mu=0 .
$$

Conversely, if the second assertion holds, then the $P_{i}$ may be regarded as commuting Markov operators defined on $L_{\infty}(X, \mathcal{F}, v d \mu)$ such that

$$
\left\|P_{i}\right\|_{L_{1}(X, \mathcal{F}, v d \mu)}=1 \quad(1 \leq i \leq d) .
$$

Thus we may apply the classical pointwise ergodic theorem for $d$-parameter semigroups of Dunford-Schwartz operators to infer that the first assertion of the lemma holds.

Proof of Theorem 3.1: (a) $\Rightarrow$ (b) and (c) $\Rightarrow$ (d) are immediate. The proof of $(b) \Rightarrow(c)$ is the same as that of the corresponding part of Theorem 2.1. (f) $\Rightarrow$ (a) follows from the classical $d$-parameter pointwise ergodic theorem, since $L_{p}(\mu) \subset L_{1}(v d \mu)$.

(d) $\Rightarrow$ (a). We may suppose that $0<U \leq 1$ on $X$. Given an $\varepsilon>0$, choose $\delta>0$ so that $\mu\{U<\delta\}<\varepsilon$. Then, since $A_{n} 1_{B} \leq 1$ on $X$ for any $B \in \mathcal{F}$, (d) implies

$$
\begin{aligned}
\int_{X}\left(A_{n(k)} \mathbf{1}_{B}\right) d \mu & \leq \delta^{-1} \int\left(A_{n(k)} \mathbf{1}_{B}\right) U d \mu+\mu\{U<\delta\} \\
& \leq \delta^{-1}\left(\int_{\left\{A_{n(k)} \mathbf{1}_{B}>t\right\}} U d \mu+t \int_{X} U d \mu\right)+\varepsilon \\
& \leq \delta^{-1} t^{-r}(\mu B)^{r / p}+\delta^{-1} t+\varepsilon \quad(t>0) .
\end{aligned}
$$


Letting $t \downarrow 0$ and then $\mu B \downarrow 0$, we see that

$$
\lim _{\mu B \rightarrow 0} \sup _{k \geq 1} \int_{B} 1 A_{n(k)} d \mu=0 .
$$

Since $\mu$ is a probability measure, it follows that the set $\left\{1 A_{n(k)}: k \geq 1\right\}$ is weakly sequentially compact in $L_{1}(\mu)$. Hence, by a mean ergodic theorem, the averages $1 A_{n}$ converge in the norm topology of $L_{1}(\mu)$ to some $v \in L_{1}^{+}(\mu)$. Then, as in the proof of Lemma 3.2, we see that $v>0$ a.e. on $X$. Therefore, regarding the $P_{i}$ as commuting Markov operators defined on $L_{\infty}(x, \mathcal{F}, v d \mu)$ such that $\left\|P_{i}\right\|_{L_{1}(X, \mathcal{F}, v d \mu)}=1$ for all $i=1,2 \ldots, d$, we can apply the classical $d$-parameter pointwise ergodic theorem to infer that for every $f$ in $L_{p}^{+}(\mu)=L_{p}^{+}\left(v^{-1} v d \mu\right)$ the limit $f^{*}(x)=\lim _{n} A_{n} f(x)$ exists a.e. on $X$ (but may equal to infinity on some subset of $X$ ) and that

$$
\left.f^{*}=E\{f \mid X, \mathcal{I}, v d \mu)\right\} \text { a.e. on } X .
$$

To see that $f^{*}<\infty$ a.e. on $X$, let us write $B=\left\{x: f^{*}(x)=\infty\right\}$. Since $B \subset \liminf _{k}\left\{A_{n(k)} f>t\right\}$ for each $t>0$, it follows from (d) together with Fatou's lemma that

$$
\int_{B} U d \mu \leq \liminf _{k} \int_{\left\{A_{n(k)} f>t\right\}} U d \mu \leq t^{-r}\left(\int_{X} f^{p} d \mu\right)^{r / p} .
$$

Letting $t \uparrow \infty$, we have $\int_{B} U d \mu=0$ and hence $\mu B=0$.

(a) $\Rightarrow$ (e). Since $L_{\infty}(\mu) \subset L_{p}(\mu)$, it follows from Lemma 3.2 that there exists a function $v_{0}$ in $L_{1}^{+}(\mu)$ such that $v_{0}>0$ a.e. on $X$ and $v_{0} P_{i}=v_{0}$ for each $i$. As before, let us regard the $P_{i}$ as commuting Markov operators on $L_{\infty}\left(X, \mathcal{F}, v_{0} d \mu\right)$. Then, considering the invertible mapping $u \rightarrow u / v_{0}$ from $L_{1}(X, \mathcal{F}, \mu)$ onto $L_{1}\left(X, \mathcal{F}, v_{0} d \mu\right)$, we see that for any $u$ in $L_{1}(\mu)$ the sequence $\left\{\left(u A_{n}\right) / v_{0}\right\}$ converges a.e. on $X$ and also in the norm topology of $L_{1}\left(v_{0} d \mu\right)$. This proves the first assertion of (e). To prove the second assertion, let $v \in L_{1}^{+}(\mu)$ be such that $v P_{i}=v$ for each $i$. Then the set $D=\{x: v(x)>0\}$ is in $\mathcal{I}$, and as before regarding the $P_{i}$ as commuting Markov operators on $L_{\infty}(D, v d \mu)$ and noticing that $L_{p}^{+}(D, \mu)=L_{p}^{+}\left(D, \frac{1}{v} v d \mu\right)$, we may apply the equivalence of (a) and (e) in Theorem 2.1 to infer the existence of a sequence $\left\{D_{N}\right\}$ of sets in $\mathcal{I}$, with $D_{N} \uparrow D$, such that if $v_{N}$ denotes the restriction function of $v$ to $D_{N}$ then

$$
v_{N}=\left(\frac{1}{v_{N}}\right)^{-1} \in L_{p^{\prime}}\left(D_{N}, \frac{1}{v} v d \mu\right)=L_{p^{\prime}}\left(D_{N}, \mu\right) .
$$


(e) $\Rightarrow$ (f) Letting $w=\lim _{n} 1 A_{n}$ in $L_{1}(\mu)$, it follows that $w P_{i}=w$ and $w>0$ a.e. on $X$. Choose $\left\{X_{N}\right\}$ in $\mathcal{I}$ so that the functions $w_{N}=w \mathbf{1}_{X_{N}}$ are in $L_{p^{\prime}}(\mu)$. It then suffices to define $v=\sum_{N=1}^{\infty} 2^{-N}\left\|w_{N}\right\|_{p^{\prime}}^{-1} w_{N}$. The proof is complete.

Theorem 3.3. Let $(X, \mathcal{F}, \mu)$ be a probability measure space and let $P_{i}(i=1,2, \ldots, d)$ be commuting Markov operators on $L_{\infty}(X, \mathcal{F}, \mu)$. Assume that each $P_{i}$ is conservative or invertible. If $1 \leq p, p_{1} \leq \infty$, then the following are equivalent.

(a) The limit $f^{*}(x)=\lim _{n} A_{n} f(x)$ exists a.e. on $X$ and $f^{*} \in L_{p_{1}}(\mu)$ for all $f$ in $L_{p}^{+}(\mu)$.

(b) For every $u$ in $L_{p_{1}^{\prime}}(\mu)$ the limit $u_{0}^{*}(x)=\lim _{n} u A_{n}(x)$ exists a.e. on $X$ and in the norm topology of $L_{1}(\mu)$; further $u_{0}^{*}$ is in $L_{p^{\prime}}(\mu)$.

(c) For every $u$ in $L_{p_{1}^{\prime}}^{+}(\mu)$ with $\|u\|_{p_{1}^{\prime}}>0$ we have

$$
0<\left\|\liminf _{n} u A_{n}\right\|_{p^{\prime}}<\infty \text {. }
$$

(d) To each $u$ in $L_{p_{1}^{\prime}}^{+}(\mu)$ there corresponds a functional $F_{u}$ defined on $L_{\infty}^{+}(\mu)$ such that

(1)

$$
\begin{cases}F_{u}\left(f_{n}\right) \rightarrow 0 & \text { whenever } f_{n} \downarrow 0 \\
F_{u}(f) \leq K_{u}\|f\|_{p}, & \begin{array}{l}
\text { a.e. on } X(\text { if } p=\infty), \\
\text { only on } u(\text { if } 1 \leq p<\infty),
\end{array}\end{cases}
$$

(2) $\begin{cases}F_{u}(t f)=t F_{u}(f) & \text { for constants } t \geq 0, \text { and } \\ F_{u}(f+g) \leq F_{u}(f)+F_{u}(g), & \end{cases}$

$$
0 \leq F_{u}(f) \leq F_{u}(f+g)
$$

$$
F_{u}\left(P_{i} f\right) \leq F_{u}(f), \text { and }
$$

(5) $\quad F_{u}(f)=\int_{X} u f d \mu$ whenever $f=P_{i} f$ for all $i=1,2, \ldots, d$. 
Proof: (a) $\Rightarrow$ (b). Since $L_{\infty}(\mu) \subset L_{p}(\mu)$, it follows from the proof of Lemma 3.2 that the sequence $\left\{\mathbf{1} A_{n}\right\}$ converges in the norm topology of $L_{1}(\mu)$ to some $v$ in $L_{1}^{+}(\mu)$ with $v P_{i}=v$ for each $P_{i}$. Since $P_{i}$ is conservative or invertible by hypothesis, it follows that $v>0$ a.e. on $X$. Hence, by the classical $d$-parameter pointwise ergodic theorem, we see that if $u \in L_{p_{1}^{\prime}}^{+}(\mu)\left(\subset L_{1}^{+}(\mu)\right)$ then the limit $u_{0}^{*}(x)=\lim _{n} u A_{n}(x)$ exists a.e. on $X$ and also in the norm topology of $L_{1}(\mu)$. In order to prove that $u_{0}^{*}$ is a function in $L_{p^{\prime}}^{+}(\mu)$, let $f$ be any function in $L_{p}^{+}(\mu)$ and put $f_{N}=f \wedge N$. Then we have

$$
\begin{aligned}
\int_{X} u_{0}^{*} f d \mu & =\lim _{N} \int_{X} u_{0}^{*} f_{N} d \mu=\lim _{N} \lim _{n}\left\langle u A_{n}, f_{N}\right\rangle \\
& =\lim _{N} \lim _{n}\left\langle u, A_{n} f_{N}\right\rangle=\lim _{N}\left\langle u, f_{N}^{*}\right\rangle \\
& \leq \int_{X} u f^{*} d \mu \leq\|u\|_{p_{1}^{\prime}}\left\|f^{*}\right\|_{p_{1}}<\infty .
\end{aligned}
$$

It follows that $u_{0}^{*} \in L_{p^{\prime}}^{+}(\mu)$.

(b) $\Rightarrow$ (c). Obvious.

(c) $\Rightarrow$ (b). There exists $v_{0} \in L_{1}^{+}(\mu)$ such that $v_{0} P_{i}=v_{0}$ for all $i=1,2, \ldots, d$ and also such that if $v \in L_{1}^{+}(\mu)$ satisfies $v P_{i}=v$ for all $i=1,2, \ldots, d$ then $\{v>0\} \subset\left\{v_{0}>0\right\}$. By virtue of (c) it is sufficient to show that $v_{0}>0$ a.e. on $X$. But, since $\left\{v_{0}=0\right\}$ is a set in $\mathcal{I}$ and the function $\tilde{u}_{0}=\liminf _{n} u A_{n}\left(u \in L_{p_{1}^{\prime}}^{+}(\mu)\right)$ satisfies $\tilde{u}_{0} P_{i}=\tilde{u}_{0} \in L_{1}^{+}(\mu)$ for all $i=1,2, \ldots, d$, this follows immediately from (c). by

(b) $\Rightarrow$ (d). For $u \in L_{p_{1}^{\prime}}^{+}(\mu)$, let $F_{u}$ be the functional on $L_{\infty}^{+}(\mu)$ defined

$$
F_{u}(f)=\int_{X} u_{0}^{*} f d \mu \quad\left(f \in L_{\infty}^{+}(\mu)\right),
$$

where $u_{0}^{*}(x)=\lim _{n} u A_{n}(x)$ a.e. on $X$. Since the sequence $\left\{u A_{n}\right\}$ converges to $u_{0}^{*}$ in the norm topology of $L_{1}(\mu), F_{u}$ satisfies all the requirements from (1) to (5).

(d) $\Rightarrow$ (a). Let $u \in L_{p_{1}^{\prime}}^{+}(\mu)$ with $u>0$ a.e. on $X$. We define a functional $q$ on $L_{\infty}(\mu)$ by putting

$$
q(f)=F_{u}\left(f^{+}\right) \quad\left(f \in L_{\infty}(\mu)\right) .
$$

Clearly we have $q(f+g) \leq q(f)+q(g), q(t f)=t q(f)$ for each constant $t \geq 0$, and $q\left(P_{i} f\right) \leq q(f)$ for each $P_{i}$. On the other hand, since $P_{i}$ is conservative or invertible by hypothesis, it follows that

$$
\left\{f \in L_{\infty}(\mu): P_{i} f=f \text { for all } i=1,2, \ldots, d\right\}=L_{\infty}(X, \mathcal{I}, \mu) .
$$


Thus, by a variant of the Hahn-Banach theorem (cf. e.g. [7, Proposition 10.5], the linear functional $f \rightarrow \tilde{F}_{u}(f)=\int_{X} u f d \mu$ defined on $L_{\infty}(X, \mathcal{I}, \mu)$ can be extended to a linear functional $F_{u}^{*}$ on $L_{\infty}(\mu)=$ $L_{\infty}(X, \mathcal{F}, \mu)$ so that

$$
F_{u}^{*}(f) \leq q(f) \text { and } F_{u}^{*}\left(P_{i} f\right)=P_{u}^{*}(f)
$$

for all $f \in L_{\infty}(\mu)$ and $P_{i}(1 \leq i \leq d) . F_{u}^{*}$ is positive, because $f \in L_{\infty}^{+}(\mu)$ implies $-F_{u}^{*}(f)=F_{u}^{*}(-f) \leq q(-f)=0$.

Now, let us set $\lambda(B)=F_{u}^{*}\left(\mathbf{1}_{B}\right)$ for $B \in \mathcal{F}$. By (1) and (6), $\lambda$ is a finite measure absolutely continuous with respect to $\mu$ and invariant under the $P_{i}$. Therefore the function $u_{0}^{*}=d \lambda / d \mu \in L_{1}^{+}(\mu)$ satisfies $u_{0}^{*} P_{i}=u_{0}^{*}$ for all $P_{i}$, and hence the set $B=\left\{x: u_{o}^{*}(x)=0\right\}$ is in $\mathcal{I}$. This and (5) imply $F_{u}^{*}\left(\mathbf{1}_{B}\right)=F_{u}\left(1_{B}\right)=\int_{B} u d \mu=0$ and $\mu B=0$. Thus the $P_{i}$ can be regarded as commuting Markov operators defined on $L_{\infty}\left(X, \mathcal{F}, u_{0}^{*} d \mu\right)$ such that

$$
\left\|P_{i}\right\|_{L_{1}\left(X, \mathcal{F}, u_{0}^{*} d \mu\right)}=1 \quad(1 \leq i \leq d) .
$$

We then apply the classical $d$-parameter pointwise ergodic theorem to infer that for any $f \geq 0$ on $X$ the pointwise limit

$$
f^{*}(x)=\lim _{n} A_{n} f(x)
$$

exists a.e. on $X$ (but may equal to infinity on some subset of $X$ ); further we see that if $0 \leq f_{n} \uparrow f$ a.e. on $X$ then $f_{n}^{*} \uparrow f^{*}$ a.e. on $X$.

Next, let $f \in L_{p}^{+}(\mu)$ be fixed arbitrarily, and put $f_{N}=f \wedge N$. Since $f_{N}^{*} \uparrow f^{*}$ a.e. on $X$ and $f_{N}^{*} \in L_{\infty}^{+}(X, \mathcal{I}, \mu)$, we see by (5), (6) and an approximation argument that

$$
\begin{aligned}
\int_{X} u f^{*} d \mu & =\lim _{N} \int_{X} u f_{N}^{*} d \mu=\lim _{N} \int_{X} u_{0}^{*} f_{N}^{*} d \mu \\
& =\lim _{N}\left(\lim _{n} \int_{X} u_{0}^{*}\left(A_{n} f_{N}\right) d \mu\right)=\lim _{N}\left(\lim _{n} \int_{X}\left(u_{0}^{*} A_{n}\right) f_{N} d \mu\right) \\
& =\lim _{N} \int_{X} u_{0}^{*} f_{N} d \mu \leq \lim _{N} F_{u}\left(f_{N}\right) \leq \lim _{N} K_{u}\left\|f_{N}\right\|_{p} \\
& \leq K_{u}\|f\|_{p}<\infty \quad\left(u \in L_{p_{1}^{\prime}}^{+}(\mu) \text { with } u>0 \text { a.e. on } X\right) .
\end{aligned}
$$

This proves that $f^{*} \in L_{p_{1}}^{+}(\mu)$, completing the proof.

Remark. Using the duality relation between Lorenz spaces $L(p, q)$ and $L\left(p^{\prime}, q^{\prime}\right)$, where $1<p, q<\infty$, it is possible to generalize Theorem 3.3 to $L(p, q)$ spaces. For this see [9], in which one-parameter semigroups of null preserving point transformations are studied.

As a direct corollary to Theorems 3.1 and 3.3 we have 
Theorem 3.4. Let $(X, \mathcal{F}, \mu)$ be a probability measure space and let $P_{i}(i=1,2, \ldots, d)$ be commuting Markov operators on $L_{\infty}(X, \mathcal{F}, \mu)$. Assume that each $P_{i}$ is conservative or invertible, and that the system $\left\{P_{1}, P_{2}, \ldots, P_{d}\right\}$ is ergodic. If $1 \leq p<\infty$, then the following are equivalent.

(a) For every $f$ in $L_{p}^{+}(\mu), \lim _{n} A_{n} f$ exists and is finite a.e. on $X$.

(b) There exists a function $v$ in $L_{p^{\prime}}^{+}(\mu)$ such that $v>0$ a.e. on $X$ and $v P_{i}=v$ for all $i=1,2, \ldots, d$.

(c) For every $u \in L_{1}^{+}(\mu)$ with $\|u\|_{1}>0$, the limit $u_{0}^{*}(x)=\lim _{n} u A_{n}(x)$ exists a.e. on $X, u_{0}^{*} \in L_{p^{\prime}}^{+}(\mu)$ and $\left\|u_{0}^{*}\right\|_{p^{\prime}}>0$.

(d) For every $u \in L_{\infty}^{+}(\mu)$ with $\|u\|_{\infty}>0$, the limit $u_{0}^{*}(x)=$ $\lim _{n} u A_{n}(x)$ exists a.e. on $X, u_{0}^{*} \in L_{p^{\prime}}^{+}(\mu)$ and $\left\|u_{0}^{*}\right\|_{p^{\prime}}>0$.

(e) There exists a functional $F$ defined on $L_{\infty}^{+}(\mu)$ such that

(8) $F(t f)=t F(f)$ for constants $t \geq 0$, and $F(f+g) \leq F(f)+F(g)$,

$$
F(1)=1 \text { and } F(f) \leq K\|f\|_{p}, K \text { being a constant, }
$$

$$
0 \leq F(f) \leq F(f+g),
$$

$$
F\left(P_{i} f\right) \leq F(f)
$$

Final remark. If we don't assume that the $P_{i}$ commute, then the equivalence of (a) and (b) in Theorem 3.4 does not hold at least for $p=1$, under the notation

$$
A_{n} f=(n+1)^{-d} \sum_{0 \leq n_{i} \leq n} P_{1}^{n_{1}} P_{2}^{n_{2}} \ldots P_{d}^{n_{d}} f
$$

We prove this by constructing a counterexample with $d=2$. Let $(X, \mathcal{F}, \mu)$ be a nonatomic probability measure space and let $\tau$ be an invertible, ergodic and measure preserving transformation on $(X, \mathcal{F}, \mu)$. Let $h$ be a function on $X$, with $h \geq 1$ on $X$, such that $h \in L_{1}(\mu)$ and $h(\log h) \notin L_{1}(\mu)$. As is well-known (see e.g. [4, p. 54], we then have

$$
\sup _{n \geq 0}(n+1)^{-1} \sum_{i=0}^{n} h \circ \tau^{i} \notin L_{1}(\mu) .
$$


On the other hand, since $\lim _{n}(n+1)^{-1} \sum_{i=0}^{n} h \circ \tau^{i}=\int_{X} h d \mu$ a.e. on $X$ and in the norm topology of $L_{1}(\mu)$, there exists a sub- $\sigma$-field $\mathcal{B}$ such that the sequence

$$
E\left\{(n+1)^{-1} \sum_{i=0}^{n} h \circ \tau^{i} \mid \mathcal{B}\right\} \quad(n \geq 0)
$$

is a.e. nonconvergent (cf. Theorem 4.3 in [1]). Here if we define

$$
P_{1} f=E\{f \mid B\} \text { and } P_{2} f=f \circ \tau \quad\left(f \in L_{\infty}(\mu)\right)
$$

then $P_{1}$ and $P_{2}$ are conservative Markov operators on $L_{\infty}(\mu)$ such that $1 P_{1}=1 P_{2}=1$. Thus (b) in Theorem 3.4 holds with $v=1$ on $X$. But (a) in Theorem 3.4 does not hold for $p=1$, because the averages

$$
A_{n} h=\frac{n}{n+1} E\left\{(n+1)^{-1} \sum_{i=0}^{n} h \circ \tau^{i} \mid \mathcal{B}\right\}+(n+1)^{-2} \sum_{i=0}^{n} h \circ \tau^{i}
$$

are a.e. nonconvergent.

\section{References}

1. DeRrienniC Y. AND Lin M., On invariant measures and ergodic theorems for positive operators, J. Functional Analysis 13 (1973), 252-267.

2. García-Cuerva J. and Rubio de Francia J. L., "Weighted Norm Inequalities and Related Topics," North-Holland, Amsterdam, 1985.

3. Garsia A. M., "Topics in Almost Everywhere Convergence," Markham, Chicago, 1970.

4. Krengel U., "Ergodic Theorems," Walter de Gruyter, Berlin, 1985.

5. MARTín-REYes F. J., On a pointwise ergodic theorem for multiparameter groups, in "Almost Everywhere Convergence," G. A. Edgar and L. Sucheston, editors, Academic Press, Boston, 1989, pp. 267-279.

6. RoKHLIN V. A., On the fundamental ideas of measure theory, Mat. Sb. (N.S.) 25 (1949), 107-150; Amer. Math. Soc. Translat. Series 1 10 (1962), 1-54.

7. Royden H. L., "Real Analysis," Macmillan, New York, 1988. 
8. SATo R., On pointwise ergodic theorems for positive operators, Studia Math. 97 (1990), 71-84.

9. SAтo R., Pointwise ergodic theorems in Lorentz spaces $L(p, q)$ for null preserving transformations, submitted for publication.

Department of Mathematics

School of Science

Okayama University

Okayama 700

JAPAN

Rebut el 4 de Març de 1994 